\title{
Resveratrol downregulates the TLR4 signaling pathway to reduce brain damage in a rat model of focal cerebral ischemia
}

\author{
JUN-RONG LEI ${ }^{1,2}$, XIAN-KUN TU ${ }^{2}$, YANG WANG ${ }^{1}$, DE-WEN TU ${ }^{2}$ and SONG-SHENG SHI ${ }^{2}$ \\ ${ }^{1}$ Department of Neurosurgery, Taihe Hospital, Hubei University of Medicine, Shiyan, Hubei 442000; \\ ${ }^{2}$ Department of Neurosurgery, Fujian Medical University Union Hospital, Fuzhou, Fujian 350001, P.R. China
}

Received June 23, 2018; Accepted November 23, 2018

DOI: $10.3892 /$ etm.2019.7324

\begin{abstract}
Previous studies have demonstrated that inflammation and disruption of the blood-brain barrier (BBB) are important pathological processes during focal cerebral ischemia. Therefore, the present study evaluated the neuroprotective effects of resveratrol against brain damage, inflammation and BBB disruption in rats with focal cerebral ischemia and assessed the potential underlying molecular mechanisms. Sprague-Dawley rats underwent cerebral ischemia/reperfusion (IR) and then received intraperitoneal resveratrol (10 and $100 \mathrm{mg} / \mathrm{kg}$ ) $2 \mathrm{~h}$ following the onset of ischemia. Following $24 \mathrm{~h}$ of ischemia, neurological deficit scores, cerebral infarctions, morphological characteristics, cerebral water content, myeloperoxidase (MPO) activity and Evans blue extravasation were assessed. Additionally, the protein expression levels of Toll-like receptor 4 (TLR4) and nuclear factor (NF)- $\kappa \mathrm{B}$ p65 were detected using western blot analyses, the mRNA expression levels of cyclooxygenase-2 (COX-2) and matrix metalloproteinase-9 (MMP-9) were examined by reverse-transcription polymerase chain reaction, and tumor necrosis factor (TNF)- $\alpha$ and interleukin (IL)- $1 \beta$ blood levels were determined by ELISA. Resveratrol significantly reduced neurological deficit scores, cerebral infarct sizes, neuronal injury, MPO activity and EB content. Cerebral ischemia increased the expression levels of TLR4, NF- $\kappa$ B p65, COX-2, MMP-9, TNF- $\alpha$ and IL-1 $\beta$, but all of these factors were reduced by resveratrol. In conclusion, the present data suggest that resveratrol reduces inflammation, $\mathrm{BBB}$ disruption and brain damage in rats following focal cerebral ischemia. Additionally, the neuroprotective effects of resveratrol against cerebral ischemia may be associated with downregulation of the TLR4 pathway.
\end{abstract}

Correspondence to: Dr Xian-Kun Tu, Department of Neurosurgery, Fujian Medical University Union Hospital, 29 Xinquan Road, Fuzhou, Fujian 350001, P.R. China

E-mail: unionnstu@hotmail.com

Key words: cerebral ischemia, inflammation, nuclear factor- $\kappa \mathrm{B}$, resveratrol, Toll-like receptor

\section{Introduction}

Brain injuries caused by transient or permanent focal cerebral ischemia develop according to a series of pathological mechanisms that include free radical release, blood-brain barrier (BBB) disruption, microglial activation, inflammation and neuronal apoptosis (1). Additionally, post-ischemic inflammation mediates the pathological processes associated with ischemic brain injury (2) Toll-like receptors (TLRs) serve a central role in innate immunity and are implicated in a range of inflammatory diseases (3). Several studies have demonstrated that TLR4 expression is elevated following cerebral ischemia (4) and that the degree of ischemic brain injury and neuroinflammation is significantly lower in TLR4-deficient mice compared with wild-type mice (5). Furthermore, nuclear factor (NF) $-\kappa \mathrm{B}$, which is a key downstream factor of the TLR4 signaling pathway, is activated following cerebral ischemia, to promote inflammatory reactions and produce inflammatory molecules that further aggravate ischemic brain injury (5-7). TLR4 signaling is a promising therapeutic target for the treatment of ischemic stroke (8) because the downregulation of TLR4 expression inhibits $\mathrm{NF}-\kappa \mathrm{B}$ and reduces the expression of inflammatory molecules; this ultimately leads to the attenuation of ischemic brain injury.

Resveratrol is a polyphenol that is abundantly expressed in a wide variety of plant species and has been reported to possess cardioprotective (9), anticancer (10), anti-inflammatory (11) and neuroprotective properties (12). Although a study demonstrated that resveratrol reduces ischemia-induced brain damage due to its anti-oxidative properties (13), its potential underlying molecular mechanisms remain unknown. Therefore, the present study investigated whether resveratrol downregulates activity in the TLR4 signaling pathway in a rat model of cerebral ischemia.

\section{Materials and methods}

Induction of ischemia-reperfusion. A total of 140 adult Sprague-Dawley rats weighing 250-300 g were obtained from the Shanghai Laboratory Animal Center (Shanghai, China). All animal experiments were approved by the Institutional Animal Care and Use Committee of Hubei University of Medicine (Shiyan, China). Rats were housed in a colony room under controlled temperature $\left(22^{\circ} \mathrm{C}\right)$, a humidity of $40-70 \%$ and 
a 12-h light/dark cycle, with free access to food and water. All surgical procedures were performed using sterile techniques in accordance with institutional guidelines. Following the induction of anesthesia with 5\% isoflurane in 70/30 medical air/oxygen, all animals were trans-orally intubated while a small rodent respirator was used to maintain adequate respiration; $3 \%$ isoflurane in 70/30 medical air/oxygen was used to maintain anesthesia.

Next, the rats were subjected to ischemia-reperfusion (IR), as described by Shi et al (14) with minor revisions. Briefly, the right common carotid artery, external carotid artery and internal carotid artery were exposed and a nylon monofilament suture with a distal cylinder (diameter: $0.32 \mathrm{~mm}$ ) was inserted from the external carotid artery into the internal carotid artery and then gently advanced to occlude the origin of the right middle cerebral artery; the suture was withdrawn $2 \mathrm{~h}$ following occlusion. In the sham-operated rats, the external carotid artery was prepared for insertion of the suture but it was not inserted. During the surgical procedure, rectal temperature was maintained at $37.0 \pm 0.5^{\circ} \mathrm{C}$ with a thermostatically controlled infrared lamp.

Experimental groups. The rats were separated into four groups as follows: i) The sham group $(n=30)$, which was subjected to the sham operation; ii) the middle cerebral artery occlusion (MCAO) group $(n=36)$, which was subjected to IR and treated with a normal saline; iii) the R10 group $(n=30)$, which was subjected to IR and treated with $10 \mathrm{mg} / \mathrm{kg}$ of resveratrol [intraperitoneal (i.p.)] the R100 group ( $\mathrm{n}=36$ ), which was subjected to IR and treated with $100 \mathrm{mg} / \mathrm{kg}$ of resveratrol (i.p.). Resveratrol was obtained from Sigma-Aldrich; Merck KGaA (Darmstadt, Germany) placed in normal saline containing 20\% hydroxypropyl $\beta$-cyclodextrin and intraperitoneally injected at $2 \mathrm{~h}$ following the onset of ischemia.

Assessment of neurological deficit scores. At $24 \mathrm{~h}$ following the cerebral IR procedure, neurological deficit scores were assessed according to the method described by Bederson et al (15) with minor revisions, as follows: $0=$ no observable deficit; $1=$ contralateral forelimb flexion; $2=$ decreased resistance to lateral push without circling; and $3=$ circling to the contralateral side.

Infarct volume analysis. At $24 \mathrm{~h}$ following the cerebral IR procedure, the animals were anesthetized and sacrificed by rapid decapitation. The brains were removed, immersed in a cold saline solution for $10 \mathrm{~min}$ and then sectioned into standard coronal slices ( $2 \mathrm{~mm}$ thick) using a brain matrix slicer. The slices were placed in the vital dye 2,3,5-triphenyltetrazolium chloride (2\% TTC; Sigma-Aldrich; Merck $\mathrm{KGaA}$ ) at $37^{\circ} \mathrm{C}$ under dark conditions for $20 \mathrm{~min}$. Following this staining procedure, infarct regions appear white, whereas non-infarct regions appear red. The infarct areas in each brain slice were measured using ImageJ software (version 1.46; National Institutes of Health, Bethesda, MD, USA) and infarct volume was calculated according to the following formula: $V=t \times\left(A_{1}+A_{2}+\ldots A_{n}\right)$, where ' $V$ ' is the infarct volume, ' $t$ ' is the slice thickness and ' $A$ ' is the infarct area.

Histopathological analysis. At $24 \mathrm{~h}$ following the cerebral IR procedure, the animals were anesthetized and perfused with
$4 \%$ paraformaldehyde. The brains were removed, fixed with $4 \%$ paraformaldehyde at $4^{\circ} \mathrm{C}$ for $24 \mathrm{~h}$ and embedded in paraffin. Next, coronal sections ( $4 \mu \mathrm{m}$ thick) were deparaffinized with xylene, rehydrated with a graded alcohol series and stained with hematoxylin and eosin (HE) at room temperature for $3 \mathrm{~min}$. The sections were visualized with a light microscope at a magnification of $\mathrm{x} 400$.

Assessment of cerebral water content. Briefly, $24 \mathrm{~h}$ following the cerebral IR procedure, the rats were sacrificed and the brains were quickly removed. The ischemic hemispheres were immediately weighed on an electronic balance to ascertain the wet weight (WW) and then dried to constant weight for $24 \mathrm{~h}$ in a $100^{\circ} \mathrm{C}$ oven to obtain the dry weight (DW). Cerebral water content was calculated using the following equation: $\mathrm{H}_{2} \mathrm{O}$ $(\%)=(\mathrm{WW}-\mathrm{DW}) / \mathrm{WW} \times 100 \%$.

Biochemical analysis. Myeloperoxidase (MPO) activity was assessed to determine the extent of inflammation. At $24 \mathrm{~h}$ following the cerebral IR procedure, the rats were anesthetized and ischemic brain samples $(1.0 \mathrm{~mm}$ from bregma to $-3.0 \mathrm{~mm}$ from bregma) were collected. MPO activity in the ischemic brain was measured with an assay kit (Nanjing Jiancheng Bioengineering Institute, Nanjing, China) according to the manufacturer's protocol; the results are expressed as U/g of tissue.

Measurement of BBB permeability. The BBB permeability was assessed using measurement of Evans blue (EB) extravasation. The EB dye ( $2 \%$ in saline, $4 \mathrm{ml} / \mathrm{kg}$ for each rat) was injected into the left jugular vein at $23 \mathrm{~h}$ following ischemia ( $1 \mathrm{~h}$ prior to sacrifice) and then the rats were transcardially perfused with PBS to remove the intravascular dye. Next, the ischemic hemispheres were homogenized in a tenfold volume of $50 \%$ trichloroacetic acid solution to precipitate the protein and centrifuged for $10 \mathrm{~min}$ at $4^{\circ} \mathrm{C}$ and $2,000 \mathrm{x}$. The resulting supernatant was diluted with ethanol (1:3) and fluorescence was measured at $610 \mathrm{~nm}$ to determine the absorbance of EB; the results are expressed as $\mu \mathrm{g} / \mathrm{g}$ of brain tissue.

Western blot analysis. At $24 \mathrm{~h}$ following the cerebral IR procedure, ischemic cortical tissue samples were collected and total protein was extracted using a protein extraction kit (Xiamen Tagene Biotechnology Co. Ltd., Xiamen, China) according to the manufacturer's protocol. Briefly, $100 \mu \mathrm{g}$ samples of protein were separated on $10 \%$ SDS polyacrylamide gels, transferred to nitrocellulose membranes and then blocked in 5\% nonfat dry milk buffer for $1 \mathrm{~h}$ at room temperature. The membranes were incubated at $4^{\circ} \mathrm{C}$ overnight with either a rabbit polyclonal antibody against TLR4 (1:1,000; cat. no. sc30002) or a mouse monoclonal antibody against NF-кB p65 (1:500; cat. no. sc71675; both Santa Cruz Biotechnology, Inc., Dallas, TX, USA) and then incubated st room temperature for $2 \mathrm{~h}$ with horseradish peroxidase-conjugated goat anti-rabbit (cat. no. PV9001) mouse (cat. no. PV9002) secondary antibodies (1:1,000; Beijing Zhongshan Jinqiao Biotechnology Co., Ltd., Beijing, China). Protein expression levels were detected with an electrochemiluminescence detection system (Dalian Meilun Biotechnology Co., Ltd., Dalian, China) and exposed on X-ray film. The densities of the protein bands were scanned 
and analyzed with an image analyzer and ImageJ software (version 1.46).

Reverse-transcription polymerase chain reaction (RT-PCR). For the RT-PCR procedure, the rats were deeply anaesthetized and transcardially perfused with ice-cold PBS. The brains were quickly removed, the cortical tissues were dissected and the samples were stored at $-80^{\circ} \mathrm{C}$ until analysis. Total RNA was extracted using TRIzol reagents (Invitrogen; Thermo Fisher Scientific, Inc.), and then reverse-transcribed at $42^{\circ} \mathrm{C}$ for $60 \mathrm{~min}$ and at $95^{\circ} \mathrm{C}$ for $5 \mathrm{~min}$ to obtain single-strand cDNA with a Reverse Transcription System (Promega Corporation, Madison, WI, USA) according to the manufacturer's protocol.

Single-strand cDNA was amplified using PCR with a $100 \mu \mathrm{l}$ reaction mixture containing $50 \mathrm{mM} \mathrm{KCl}, 10 \mathrm{mM}$ Tris- $\mathrm{HCl}$ ( $\mathrm{pH}$ 9.0), $2 \mathrm{mM} \mathrm{MgCl}{ }_{2}, 200 \mu \mathrm{M}$ dNTPs, $0.5 \mu \mathrm{M}$ of sense and antisense primers, and 2.5 units of Taq DNA polymerase (Promega Corporation). The primer sequences were as follows: Cyclooxygenase-2 (COX-2; sense: 5'-CCA TGTCAAAACCGTGGTGAATG-3'; antisense: 5'-ATGGGA GTTGGGCAGTCATCAG-3'; product size: 374 bp), matrix metalloproteinase-9 (MMP-9; sense: 5'-AAGGATGGTCTA CTGGCAC-3'; antisense: 5'-AGAGATTCTCACTGGGGC-3'; product size: $279 \mathrm{bp}$ ) and the internal standard $\beta$-actin, (sense: 5'-CCCATCTATGAGGGTTACGC-3'; antisense: 5'-TTTAAT GTCACGCACGATTTC-3'; product size: $150 \mathrm{bp}$ ). The reactions were initially heated at $94^{\circ} \mathrm{C}$ for $4 \mathrm{~min}$ and then at $94^{\circ} \mathrm{C}$ for $40 \mathrm{sec}, 58^{\circ} \mathrm{C}$ for $40 \mathrm{sec}$, and $72^{\circ} \mathrm{C}$ for $50 \mathrm{sec}$ over a total of 38 cycles. The reactions were stopped at $72^{\circ} \mathrm{C}$ for $7 \mathrm{~min}$. The PCR products $(10 \mu \mathrm{l})$ were electrophoresed in a $2 \%$ agarose gel containing ethidium bromide and DNA band optical density was measured with a UVP gel analysis system (Quantity One; Bio-Rad Laboratories, Inc., Hercules, CA, USA).

ELISAs. At $24 \mathrm{~h}$ following the cerebral IR procedure, $1 \mathrm{ml}$ blood samples were drawn from the rat hearts. The samples were centrifuged at $4^{\circ} \mathrm{C}$ and $2,000 \mathrm{x}$ g for $10 \mathrm{~min}$ and then the supernatants were collected. The plasma contents of tumor necrosis factor- $\alpha$ (TNF- $\alpha$ ) and interleukin (IL)- $1 \beta$ were measured using rat TNF- $\alpha$ (cat. no. RTA00) or IL-1 $\beta$ (cat. no. RLB00) ELISA kits (R\&D Systems, Inc., Minneapolis, MN, USA).

Statistical analysis. SPSS 13.0 software (SPSS, Inc., Chicago, IL, USA) was used to analyze the data in the current study. All results are presented as the mean \pm standard deviation. All statistical analyses were performed using analysis of variance followed by Student-Newman-Keuls test for multiple group comparisons. $\mathrm{P}<0.05$ was considered to indicate a statistically significant difference.

\section{Results}

Resveratrol reduces neurological deficit scores, cerebral infarct size, neuronal injury and brain edema. Rats subjected to the cerebral IR procedure exhibited increases in neurological deficit scores, cerebral infarct size, neuronal injury and cerebral water content. Although there were no differences between the vehicle-treated group and the $10 \mathrm{mg} / \mathrm{kg}$ resveratrol group, $100 \mathrm{mg} / \mathrm{kg}$ of resveratrol significantly reduced
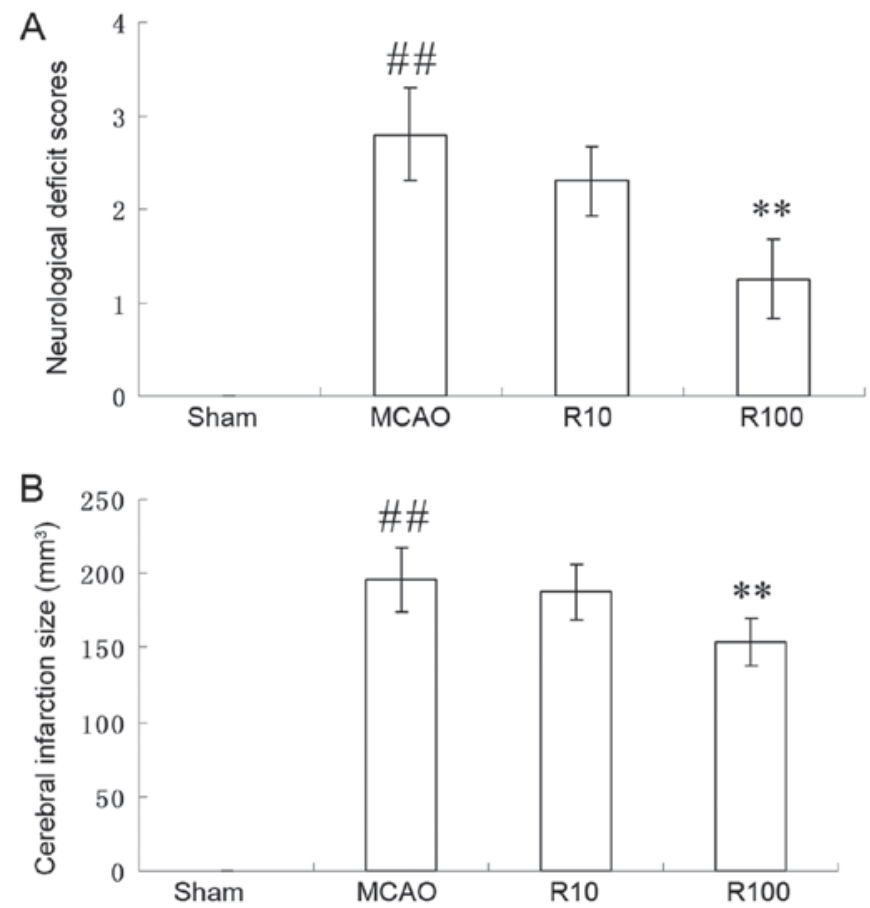

Figure 1. Resveratrol reduces neurological deficit scores and cerebral infarct volume following cerebral IR. (A) Neurological deficit scores were significantly increased at $24 \mathrm{~h}$ following cerebral IR $(2.8 \pm 0.5)$ but exhibited significant reductions following treatment with $100 \mathrm{mg} / \mathrm{kg}$ of resveratrol (1.25 \pm 0.42$)$, but not $10 \mathrm{mg} / \mathrm{kg}$ of resveratrol (2.3 \pm 0.37$)$. (B) Cerebral infarct volume was significantly larger at $24 \mathrm{~h}$ following cerebral IR $(195.4 \pm 21.5)$, but was significantly reduced following treatment with $100 \mathrm{mg} / \mathrm{kg}$ of resveratrol $(153.8 \pm 15.4) ; \mathrm{n}=12$ in each group; ${ }^{\# \prime} \mathrm{P}<0.01$ vs. the sham operation group; ${ }^{* *} \mathrm{P}<0.01$ vs. the MCAO group. R10, resveratrol $10 \mathrm{mg} / \mathrm{kg}$; R100, resveratrol $100 \mathrm{mg} / \mathrm{kg}$; MCAO, middle cerebral artery occlusion; IR, ischemia/reperfusion.

the neurological deficit scores $(\mathrm{P}<0.01$; Fig. 1A) and cerebral infarct size $(\mathrm{P}<0.01$; Fig. $1 \mathrm{~B})$ at $24 \mathrm{~h}$ following cerebral IR. Additionally, HE staining revealed that no injured neurons were identified in the sham-operated group (Fig. 2A), cerebral IR caused neuronal injury in the ischemic hemisphere (Fig. 2B). Although $10 \mathrm{mg} / \mathrm{kg}$ of resveratrol (Fig. 2C) did not reduce the extent of neuronal injury, $100 \mathrm{mg} / \mathrm{kg}$ of resveratrol did (Fig. 2D). Furthermore, $100 \mathrm{mg} / \mathrm{kg}$ of resveratrol significantly reduced the cerebral water content in the ischemic hemisphere produced by cerebral IR when compared with the MCAO group ( $\mathrm{P}<0.01$; Fig. 3$)$.

Resveratrol reduces ischemia-induced inflammation. MPO enzymatic activity was assessed to determine the extent of inflammation in ischemic brain tissues. The experimental data demonstrated that MPO activity was significantly elevated at $24 \mathrm{~h}$ following cerebral IR compared with the sham-operated group $(\mathrm{P}<0.05)$. However, this elevation exhibited a significant decrease following treatment with $100 \mathrm{mg} / \mathrm{kg}$ of resveratrol $(\mathrm{P}<0.01)$ but not $10 \mathrm{mg} / \mathrm{kg}$ of resveratrol ( $\mathrm{P}>0.05$; Fig. 4A).

Resveratrol reduces $B B B$ permeability. The EB extravasation analyses were performed to assess the extent of BBB permeability. The experimental data demonstrated that the EB content was significantly elevated at $24 \mathrm{~h}$ following cerebral IR compared with the sham operation group $(\mathrm{P}<0.05)$. However, 

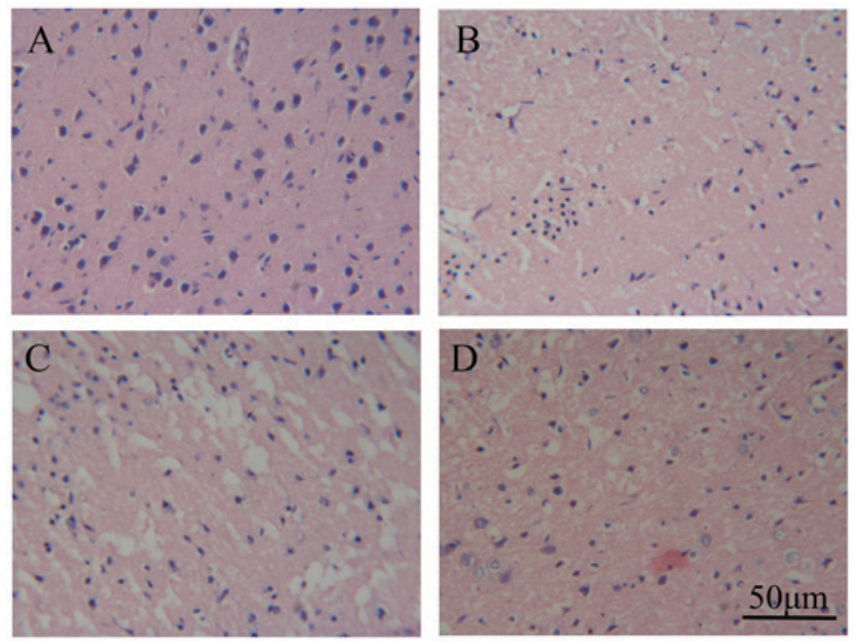

Figure 2. Resveratrol reduces ischemic neuronal injury following cerebral IR There were no neuronal injuries in the (A) sham-operated group. Ischemic neuronal injury increased in the (B) ischemic group but not by (C) $10 \mathrm{mg} / \mathrm{kg}$ of resveratrol. Ischemic neuronal injury was reduced by (D) $100 \mathrm{mg} / \mathrm{kg}$ of resveratrol. Scale bar $=50 \mu \mathrm{m}$. IR, ischaemia/reperfusion.
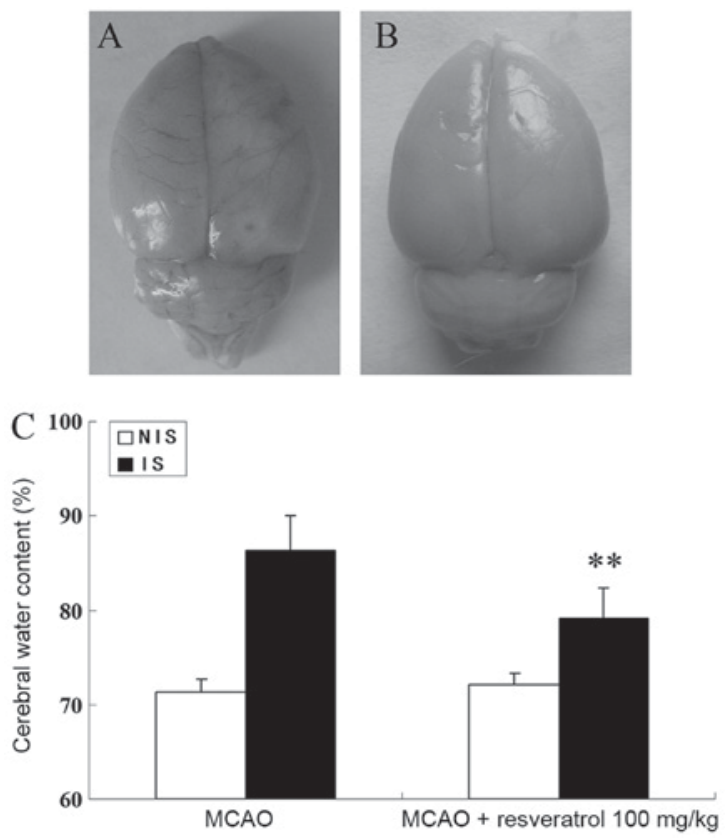

Figure 3. Resveratrol reduces cerebral water content following cerebral IR. Representative images of rat brains from (A) a MCAO rat and (B) a $100 \mathrm{mg} / \mathrm{kg}$ resveratrol-treated rat. (C) Quantification of cerebral water content. Cerebral water content was significantly elevated at $24 \mathrm{~h}$ following cerebral IR $(86.43 \pm 3.61 \%)$ but was significantly reduced by $100 \mathrm{mg} / \mathrm{kg}$ of resveratrol (79.25 $\pm 3.13 \%) ; \mathrm{n}=6 ;{ }^{* *} \mathrm{P}<0.01$ vs. the MCAO group. NIS, non-ischemic brain IS, ischemic brain; IR, ischaemia/reperfusion; MCAO, middle cerebral artery occlusion.

this elevation exhibited a significant decrease following treatment with $100 \mathrm{mg} / \mathrm{kg}$ of resveratrol $(\mathrm{P}<0.01)$ but not $10 \mathrm{mg} / \mathrm{kg}$ of resveratrol ( $\mathrm{P}>0.05$; Fig. 4B).

Resveratrol downregulates the protein expression levels of TLR4 and NF- $\kappa B$. The protein expression levels of TLR4 and $\mathrm{NF}-\kappa \mathrm{B}$ p65 in ischemic brain tissues increased at $24 \mathrm{~h}$ following cerebral IR but were significantly downregulated by resveratrol $(\mathrm{P}<0.05$; Fig. 5).
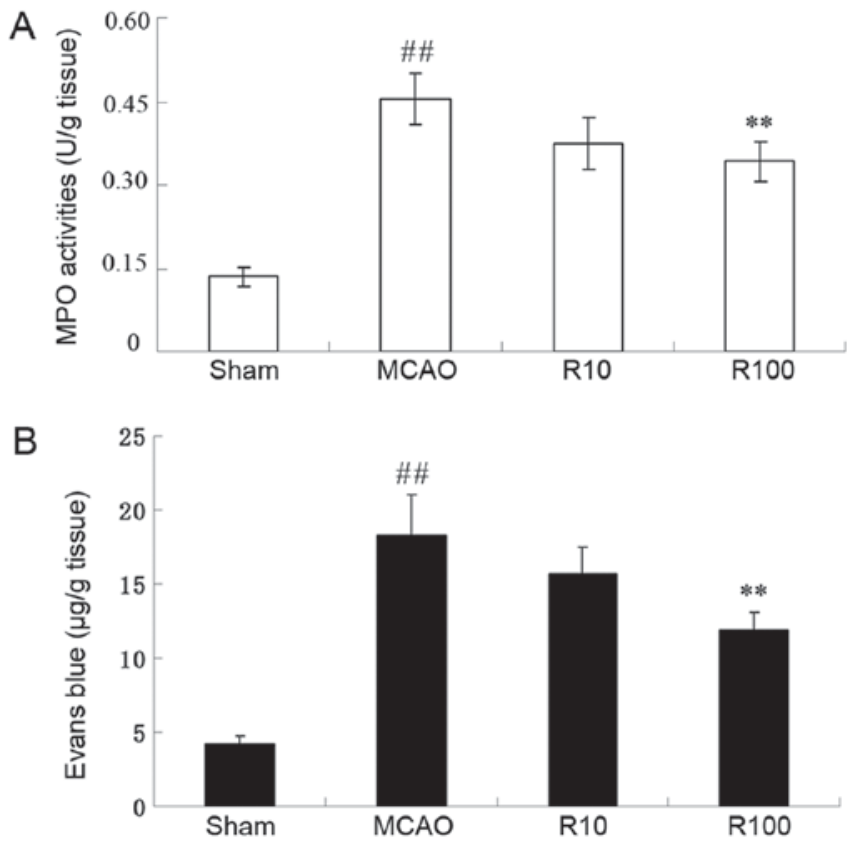

Figure 4. Resveratrol effect on cerebral inflammation and permeability. Resveratrol reduces (A) MPO activity and (B) BBB permeability following cerebral IR. MPO content was significantly elevated at $24 \mathrm{~h}$ following cerebral IR $(0.455 \pm 0.046)$ but was significantly reduced by $100 \mathrm{mg} / \mathrm{kg}$ of resveratrol $(0.342 \pm 0.035) ; \mathrm{n}=12$. Evans blue content was increased at $24 \mathrm{~h}$ following cerebral IR $(18.3 \pm 2.7)$ but was significantly reduced by $100 \mathrm{mg} / \mathrm{kg}$ of resveratrol $(11.9 \pm 1.2) ; \mathrm{n}=5$ in each group; ${ }^{\#} \mathrm{P}<0.01$ vs. the sham operation group; ${ }^{* *} \mathrm{P}<0.01$ vs. MCAO group. R10, resveratrol $10 \mathrm{mg} / \mathrm{kg}$; R100, Resveratrol $100 \mathrm{mg} / \mathrm{kg}$; MPO, myeloperoxidase; IR, ischaemia/reperfusion; $\mathrm{MCAO}$, middle cerebral occlusion artery; BBB, blood-brain barrier.

Resveratrol downregulates the $m R N A$ expression levels of COX-2 and MMP-9. The mRNA expression levels of COX-2 and MMP-9 mRNA in ischemic brain tissues increased at $24 \mathrm{~h}$ following cerebral IR but were significantly downregulated by resveratrol $(\mathrm{P}<0.01$; Fig. 6).

Resveratrol reduces blood levels of TNF- $\alpha$ and $I L-1 \beta$. The blood levels of TNF- $\alpha$ and IL-1 $\beta$ increased at $24 \mathrm{~h}$ following cerebral IR but were significantly reduced by resveratrol $(\mathrm{P}<0.05$; Fig. 7).

\section{Discussion}

Stroke remains a leading cause of mortality and neurological disabilities in adults around the world. Currently, acute ischemic stroke (AIS) is treated using two major therapeutic strategies: Thrombolytic therapy and neuroprotective therapy (16) Although tissue-plasminogen activator is the only FDA-approved therapy for AIS within a 3-4.5 h time window (17), only $1-2 \%$ of patients have the opportunity to receive thrombolytic therapy due to the brevity of this window (18). Additionally, reperfusion followed by thrombolysis may exacerbate brain injuries via a series of pathological mechanisms including inflammation and BBB disruption (19). Therefore, identification of novel potential neuroprotective agents targeting the pathological mechanisms underlying cerebral IR is necessary.

The present study demonstrated that resveratrol protected brains against ischemic stroke in an experimental rat model, 
A
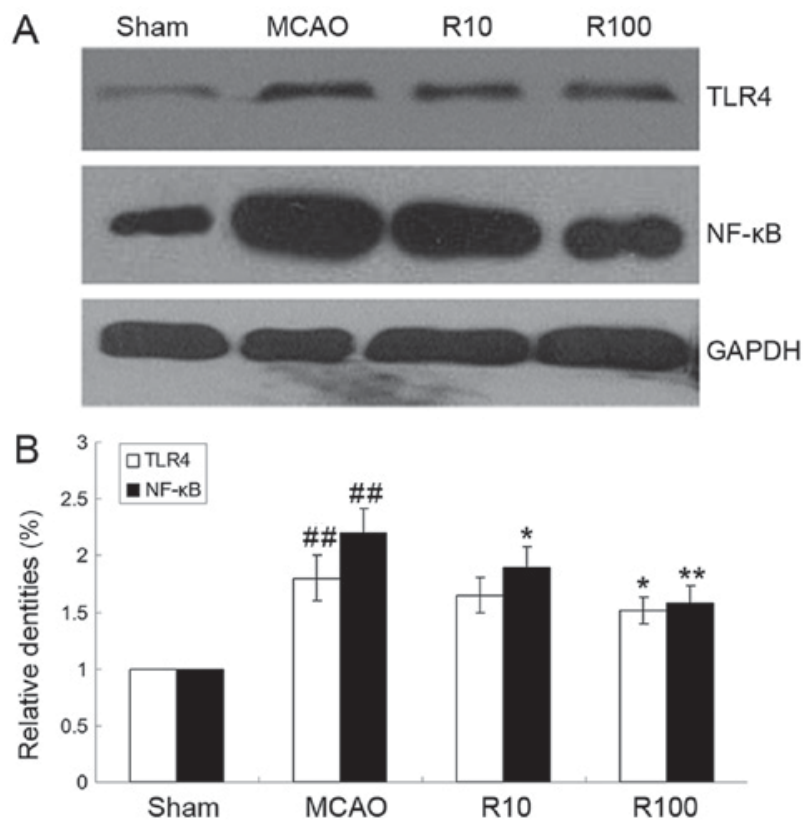

Figure 5. Resveratrol downregulates the expression levels of TLR4 and NF-kB p65 following cerebral IR. Representative protein expression bands from western blot analyses of (A) TLR4 and NF- $\mathrm{kB}$ p65. (B) Summary of the experimental data; $\mathrm{n}=12$ in each group; ${ }^{\# \#} \mathrm{P}<0.01$ vs. the sham operation group; " $\mathrm{P}<0.05$ and ${ }^{* *} \mathrm{P}<0.01$ vs. the MCAO group. R10, resveratrol $10 \mathrm{mg} / \mathrm{kg}$; $\mathrm{R} 100$, resveratrol $100 \mathrm{mg} / \mathrm{kg}$; NF- $\mathrm{kB}$, nuclear factor- $\mathrm{\kappa} B$; IR, ischaemia reperfusion; TLR, toll-like receptor; MCAO, middle cerebral occlusion artery.

A
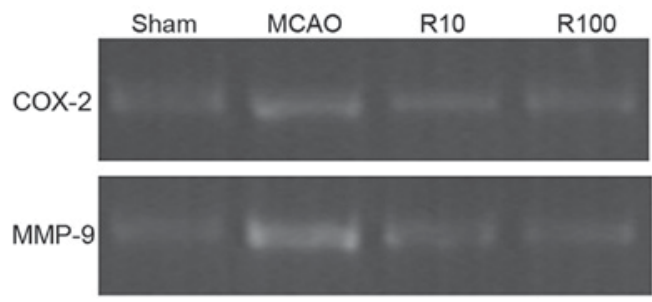

$\beta$-actin
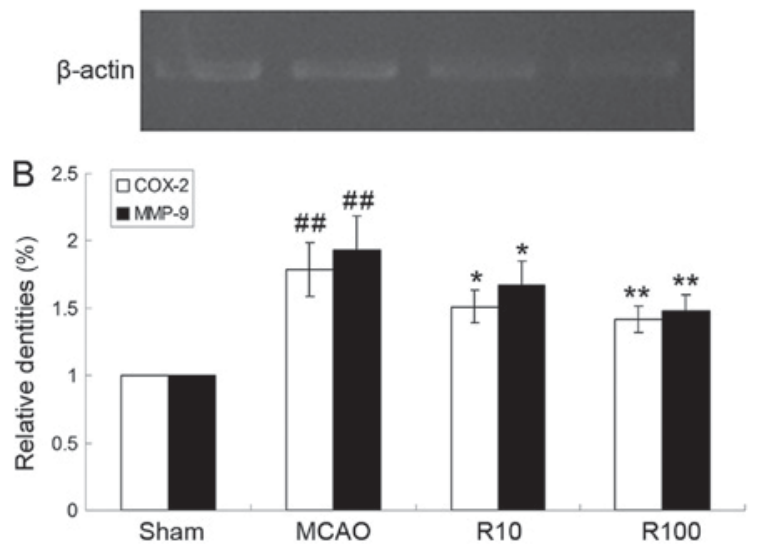

Figure 6. Resveratrol downregulates the expression levels of COX-2 and MMP-9 following cerebral ischaemia reperfusion. (A) Representative mRNA expression bands of COX-2 and MMP-9. (B) Summary of the experimental data; $\mathrm{n}=12$ in each group, ${ }^{\# \#} \mathrm{P}<0.01$ vs. the sham operation group; ${ }^{*} \mathrm{P}<0.05$ and ${ }^{* *} \mathrm{P}<0.01$ vs. the MCAO group. R10, resveratrol $10 \mathrm{mg} / \mathrm{kg}$; R100, resveratrol $100 \mathrm{mg} / \mathrm{kg}$; MMP, matrix metalloproteinase; COX, cyclooxygenase; MCAO, middle cerebral artery occlusion.

which is consistent with a previous study (20). Several studies have investigated the potential mechanisms underlying the neuroprotective capabilities of resveratrol. For example,

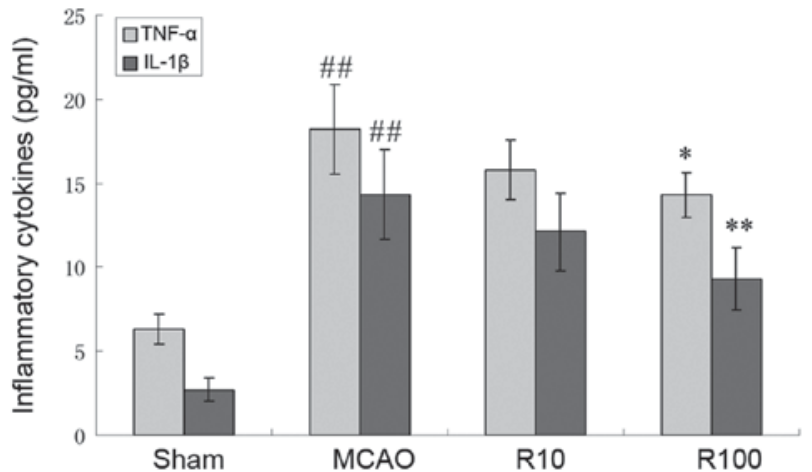

Figure 7. Resveratrol decreases the expression levels of TNF- $\alpha$ and IL-1 $\beta$ following cerebral IR. Blood levels of proinflammatory cytokines (TNF- $\alpha$ and IL-1 $\beta$ ) were significantly increased at $24 \mathrm{~h}$ following cerebral IR but were significantly reduced by resveratrol; $\mathrm{n}=12$ in each group; ${ }^{\# \#} \mathrm{P}<0.01$ vs. the sham operation group; ${ }^{*} \mathrm{P}<0.05$ and ${ }^{* *} \mathrm{P}<0.01$ vs. the MCAO group. $\mathrm{R} 10$, resveratrol $10 \mathrm{mg} / \mathrm{kg}$; R100, resveratrol $100 \mathrm{mg} / \mathrm{kg}$; MCAO, middle cerebral artery occlusion; IR, ischaemia/reperfusion; IL, interleukin; TNF, tumor necrosis factor.

Sinha et al (21) demonstrated that resveratrol protects rat brain tissues against focal cerebral ischemia by reducing oxidative stress, while Tsai et al (22) demonstrated that resveratrol downregulates the expression of inducible nitric oxide synthase (NOS) and upregulates the expression of endothelial NOS, which may explain how resveratrol protects rat brains against focal cerebral ischemia. Additionally, Li et al (23) reported that resveratrol attenuates ischemic brain injury and that these effects may be associated with the inhibition of neuronal apoptosis via the upregulation of hippocampal Bcl-2.

Another previous study demonstrated that resveratrol is neuroprotective against cerebral ischemia injury via anti-oxidant and anti-inflammatory mechanisms (24). Although specific reports demonstrated that resveratrol does not have beneficial effects on memory and cognitive dysfunction (25), these differences may be attributed to the use of different disease models. Therefore, additional studies will be required to clarify these discrepancies. The present study demonstrated that resveratrol decreased the enzymatic activity of MPO, which suggests that resveratrol possesses anti-inflammatory properties that are beneficial following ischemic stroke. It is also possible that the neuroprotective effects of resveratrol against ischemic stroke may be associated with its anti-inflammatory activities.

TLRs serve critical roles in the induction of innate and adaptive immunity (26). Additionally, these receptors possess leucine-rich repeats in their extracellular region, which are responsible for the recognition of pathogen-associated molecular patterns and endogenous 'danger'-associated molecular patterns and a Toll IL-1 receptor domain in the intracellular region that is required for the initiation of intracellular signaling (27). Of the TLR family, TLR4 has been of particular interest because it is the primary receptor recognizing bacterial infections and endogenous ligands released following tissue injury. Endogenous 'danger signals', including high mobility group box protein 1 and heat shock proteins activate TLR4 signaling. The activation of TLR4 stimulates IкB- $\alpha$ phosphorylation and degradation, which results in the nuclear

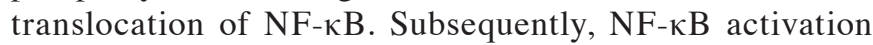


regulates the expression levels of inflammatory genes that are involved in innate immune responses and lead to the initiation of inflammation (28).

The TLR4 and NF- $\mathrm{BB}$ signaling pathways are widely considered to mediate ischemic brain injury processes and to be a promising therapeutic target for ischemic stroke $(29,30)$. Neurological dysfunction scores and cerebral infarction scores in TLR4-deficient mice are significantly lower compared with those of wild-type mice, and the present study demonstrated that resveratrol downregulated the expression of TLR4 and inhibited the activation of NF- $\mathrm{BB}$. The low expression of TLR4 following resveratrol administration could weaken activation of the TLR4/NF- $\mathrm{BB}$ signaling pathway and attenuate inflammation, which in turn would reduce the ischemic brain injury induced by inflammation.

Zhao et al (31) reported that ischemic stroke leads to enhanced expression of COX-2, which results in progressive ischemic brain injury. Nimesulide, a selective COX-2 inhibitor, attenuates COX-2 activity and ameliorates cerebral ischemia injury (32), which indicates that COX-2 may be an important therapeutic target for AIS. MMP-9, a proteolytic enzyme, degrades important structures in the microvascular wall to increase microvascular permeability and BBB disruption (33). During cerebral ischemia, MMP-9 expression is upregulated, which leads to the degradation of occludin that, in turn, causes BBB leakage, brain edema, brain hemorrhages and secondary brain damage (34). In the present study, resveratrol downregulated the mRNA expression levels of COX-2 and MMP-9 following cerebral IR in rats; this suggests that resveratrol attenuated inflammatory reactions and $\mathrm{BBB}$ disruption, and furthermore that these activities may be associated with the inhibition of COX-2 and MMP-9.

During cerebral ischemia, the TLR4/NF- $\kappa \mathrm{B}$ pathway regulates the expression levels of inflammatory cytokines, including TNF- $\alpha$ and IL-1 $\beta$, which propagates the inflammatory cascade reaction and eventually increases brain damage. The blood levels of TNF- $\alpha$ and IL-1 $\beta$ are increased following cerebral ischemia, but are decreased by curcumin (29). In the present study, the blood levels of TNF- $\alpha$ and IL- $1 \beta$ were elevated, but were lowered by resveratrol treatment. These findings suggest that the anti-inflammatory properties of resveratrol may be attributable to inhibition of the TLR4/NF- $\mathrm{KB}$ signaling pathway.

In conclusion, the present study demonstrated that resveratrol reduced neurological dysfunction, neuronal injury, cerebral infarction and BBB permeability in a rat model of focal cerebral ischemia, and demonstrated that these activities may be associated with the downregulation of inflammatory processes and the TLR4 signaling pathway. These experimental results suggest that the TLR4 signaling pathway may be an important therapeutic target for and resveratrol a promising neuroprotective agent against, ischemic stroke.

\section{Acknowledgements}

The authors would like to give thanks for the technical assistance of Professor Dong-Sheng Li from the Institute of Life Science at Taihe Hospital, Hubei University of Medicine (Shiyan, China).

\section{Funding}

The Natural Science Foundation of the Fujian Province of China (grant no. 2017J01204) and the Key Young Talents Cultivation Project of Health and Family Planning Commission of the Fujian Province of China (grant no. 2016-ZQN-28) contributed to this study.

\section{Availability of data and materials}

All data generated or analyzed during this study are included in this article.

\section{Authors' contributions}

JRL and XKT designed the study and wrote the paper. JRL, YW, DWT and SSS performed the experiments and helped perform the analysis with constructive discussions. XKT revised the manuscript. All authors read and approved the manuscript.

\section{Ethics approval and consent to participate}

All animal experiments were approved by the Institutional Animal Care and Use Committee of Hubei University of Medicine (Shiyan, China).

\section{Patient consent for publication}

Not applicable.

\section{Competing interests}

The authors declare that they have no competing interests.

\section{References}

1. Dirnagl U: Pathobiology of injury after stroke: The neurovascular unit and beyond. Ann N Y Acad Sci 1268: 21-25, 2012.

2. Gu Y, Chen J and Shen J: Herbal medicines for ischemic stroke: Combating inflammation as therapeutic targets. J Neuroimmune Pharmacol 9: 313-339, 2014.

3. Lucas K and Maes M: Role of the toll like receptor (TLR) radical cycle in chronic inflammation: Possible treatments targeting the TLR4 pathway. Mol Neurobiol 48: 190-204, 2013.

4. Tu XK, Yang WZ, Shi SS, Wang CH, Zhang GL, Ni TR, Chen CM, Wang R, Jia JW and Song QM: Spatio-temporal distribution of inflammatory reaction and expression of TLR2/4 signaling pathway in rat brain following permanent focal cerebral ischemia. Neurochem Res 35: 1147-1155, 2010.

5. Caso JR, Pradillo JM, Hurtado O, Lorenzo P, Moro MA and Lizasoain I: Toll-like receptor 4 is involved in brain damage and inflammation after experimental stroke. Circulation 115: 1599-1608, 2007.

6. Tu XK, Yang WZ, Shi SS, Chen Y, Wang CH, Chen CM and Chen Z: Baicalin inhibits TLR2/4 signaling pathway in rat brain following permanent cerebral ischemia. Inflammation 34: 463-470, 2011.

7. Guan T, Liu Q, Qian Y, Yang H,Kong J,Kou J and Yu B: Ruscogenin reduces cerebral ischemic injury via NF- $\mathrm{KB}$-mediated inflammatory pathway in the mouse model of experimental stroke. Eur J Pharmacol 714: 303-311, 2013.

8. Lan L, Tao J, Chen A, Xie G, Huang J, Lin J, Peng J and Chen L: Electroacupuncture exerts anti-inflammatory effects in cerebral ischemia-reperfusion injured rats via suppression of the TLR4/NF-кB pathway. Int J Mol Med 31: 75-80, 2013.

9. Mokni M, Hamlaoui S, Karkouch I, Amri M, Marzouki L, Limam F and Aouani E: Resveratrol provides cardioprotection after ischemia/reperfusion injury via modulation of antioxidant enzyme activities. Iran J Pharm Res 12: 867-875, 2013. 
10. Kma L: Synergistic effect of resveratrol and radiotherapy in control of cancers. Asian Pac J Cancer Prev 14: 6197-6208, 2013.

11. Taguchi A, Wada-Hiraike O, Kawana K, Koga K, Yamashita A Shirane A, Urata Y, Kozuma S, Osuga Y and Fujii T: Resveratrol suppresses inflammatory responses in endometrial stromal cells derived from endometriosis: A possible role of the sirtuin 1 pathway. J Obstet Gynaecol Res 40: 770-778, 2014.

12. Zhou XM, Zhou ML, Zhang XS, Zhuang Z, Li T, Shi JX and Zhang X: Resveratrol prevents neuronal apoptosis in an early brain injury model. J Surg Res 189: 159-165, 2014.

13. Ren J, Fan C, Chen N, Huang J and Yang Q: Resveratrol pretreatment attenuates cerebral ischemic injury by upregulating expression of transcription factor $\mathrm{Nrf} 2$ and $\mathrm{HO}-1$ in rats. Neurochem Res 36: 2352-2362, 2011.

14. Shi SS, Yang WZ, Tu XK, Wang CH, Chen CM and Chen Y: 5-Lipoxygenase inhibitor zileuton inhibits neuronal apoptosis following focal cerebral ischemia. Inflammation 36: 1209-1217, 2013.

15. Bederson JB, Pitts LH, Tsuji M, Nishimura MC, Davis RL and Bartkowski H: Rat middle cerebral artery occlusion: Evaluation of the model and development of a neurologic examination. Stroke 17: 472-476, 1986.

16. Chen F, Qi Z, Luo Y, Hinchliffe T, Ding G, Xia Y and Ji X Non-pharmaceutical therapies for stroke: Mechanisms and clinical implications. Prog Neurobiol 115: 246-269, 2014.

17. Salam KA, Ummer K, Pradeep Kumar VG and Noone ML: Intravenous thrombolysis for acute ischemic stroke in the 3 to 4.5-hour window-The Malabar experience. Int J Stroke 9: 426-428, 2014

18. Bansal S, Sangha KS and Khatri P: Drug treatment of acute ischemic stroke. Am J Cardiovasc Drugs 13: 57-69, 2013.

19. Pan J, Konstas AA, Bateman B, Ortolano GA and Pile-Spellman J: Reperfusion injury following cerebral ischemia: Pathophysiology, MR imaging, and potential therapies. Neuroradiology 49: 93-102, 2007.

20. Lu KT, Chiou RY, Chen LG, Chen MH, Tseng WT, Hsieh HT and Yang YL: Neuroprotective effects of resveratrol on cerebral ischemia-induced neuron loss mediated by free radical scavenging and cerebral blood flow elevation. J Agric Food Chem 54: 3126-3131, 2006.

21. Sinha K, Chaudhary G and Gupta YK: Protective effect of resveratrol against oxidative stress in middle cerebral artery occlusion model of stroke in rats. Life Sci 71: 655-665, 2002.

22. Tsai SK, Hung LM, Fu YT, Cheng H, Nien MW, Liu HY, Zhang FB and Huang SS: Resveratrol neuroprotective effects during focal cerebral ischemia injury via nitric oxide mechanism in rats. J Vasc Surg 46: 346-353, 2007.
23. Li Z, Pang L, Fang F, Zhang G, Zhang J, Xie M and Wang L: Resveratrol attenuates brain damage in a rat model of focal cerebral ischemia via up-regulation of hippocampal Bcl-2. Brain Res 1450: 116-124, 2012.

24. Orsu P, Murthy BV and Akula A: Cerebroprotective potential of resveratrol through anti-oxidant and anti-inflammatory mechanisms in rats. J Neural Transm (Vienna) 120: 1217-1223, 2013.

25. Farzaei MH, Rahimi R, Nikfar S and Abdollahi M: Effect of resveratrol on cognitive and memory performance and mood: A meta-analysis of 225 patients. Pharmacol Res 128: 338-344, 2018.

26. Ulevitch RJ: Therapeutics targeting the innate immune system. Nat Rev Immunol 4: 512-520, 2004.

27. Means TK, Golenbock DT and Fenton MJ: The biology of Toll-like receptors. Cytokine Growth Factor Rev 11: 219-232, 2000.

28. Carmody RJ and Chen YH: Nuclear factor-kappaB: Activation and regulation during toll-like receptor signaling. Cell Mol Immunol 4: 31-41, 2007.

29. Tu XK, Yang WZ, Chen JP, Chen Y, Ouyang LQ, Xu YC and Shi SS: Curcumin inhibits TLR2/4-NF- $\mathrm{KB}$ signaling pathway and attenuates brain damage in permanent focal cerebral ischemia in rats. Inflammation 37: 1544-1551, 2014.

30. Xiang HF, Cao DH, Yang YQ, Wang HQ, Zhu LJ, Ruan BH, Du J and Wang MC: Isoflurane protects against injury caused by deprivation of oxygen and glucose in microglia through regulation of the Toll-like receptor 4 pathway. J Mol Neurosci 54: 664-670, 2014

31. Zhao Y, Patzer A, Herdegen T, Gohlke P and Culman J: Activation of cerebral peroxisome proliferator-activated receptors gamma promotes neuroprotection by attenuation of neuronal cyclooxygenase- 2 overexpression after focal cerebral ischemia in rats. FASEB J 20: 1162-1175, 2006.

32. Candelario-Jalil E, González-Falcón A, Garcia-Cabrera M, León OS and Fiebich BL: Wide therapeutic time window for nimesulide neuroprotection in a model of transient focal cerebral ischemia in the rat. Brain Res 1007: 98-108, 2004.

33. Wei H, Wang S, Zhen L, Yang Q, Wu Z, Lei X, Lv J, Xiong L and Xue R: Resveratrol attenuates the blood-brain barrier dysfunction by regulation of the MMP-9/TIMP-1 balance after cerebral ischemia reperfusion in rats. J Mol Neurosci 55: 872-879, 2015.

34. Wang L, Li Z, Zhang X, Wang S, Zhu C, Miao J, Chen L, Cui L and Qiao H: Protective effect of shikonin in experimental ischemic stroke: Attenuated TLR4, p-p38MAPK, NF- $k B$, TNF- $\alpha$ and MMP-9 expression, up-regulated claudin-5 expression, ameliorated BBB permeability. Neurochem Res 39: 97-106, 2014. 